\section{Potential Welfare and the Sum of \\ Individual Compensating or Equivalent Variations*}

J. Ruiz-Castillo

Departamento de Teoria Económica

Universidad Complutense de Madrid, Pozuelo, Madrid-28023, Spain

We investigate under which conditions the sign of the sum of both the individual 作 welfare. Our results reproduce exactly those of Chipman and Moore for the National Income Test, which did not rely on individual measures of welfare directly related to consumers' preferences:

(i) a necessary and sufficient condition for the new test to be valid is that individual preferences are identical and homothetic; and

(ii) if the distribution of income is constant and preferences are homothetic Cest is valid only if preferences are also identical. Journal of Economic Literature Classification Numbers: 021, 022, 024 .

\section{INTRODUCTION}

Suppose we want to compare from the social point of view, but using only observable concepts, the outcome of the economic process in two different situations in the simplest possible case; namely, abstracting from the conceptual and practical problems which plague the measurement of national income in the presence of external effects, imperfect competition, the activities of the public sector, and other complicating factors.

Consider then two situations characterized by a vector of prices and an aggregate commodity bundle $\left(p^{t}, y^{t}\right), t=1,2$, with $y^{2} \neq y^{1}$, arising from a pair of competitive equilibria in a sense made precise below. We say that real national income has risen in situation 2 (relative to situation 1 ) according to the:

${ }^{*}$ I have greatly benefited from numerous comments and suggestions by an anonymous referee. Any remaining shortcomings are my sole responsibility. (a) Laspeyres criterion, if and only if $p^{1} \cdot y^{2} \geqslant p^{1} \cdot y^{1}$;

(b) Paasche criterion, if and only if $p^{2} \cdot y^{2} \geqslant p^{2} \cdot y^{1}$.

In a world of several individuals, it is most likely that both situation will be noncomparable according to the Pareto criterion. Thus, following the ideas put forth in the Compensation Principle literature, let us judge social welfare in terms of what has been called the Kaldor-HicksSamuelson (KHS) ordering: given any two aggregate bundles $y$ and $y^{\prime}, y$ is said to afford a higher potential welfare than $y^{\prime}$, if for any allocation of $y^{\prime}$ among the individuals, there exists an allocation of $y$ which is Pareto superior to it. Whatever the shortcomings of this relation as a basis for passing social judgments, it is at least free from contradictions and has been shown to be reflexive and transitive (see Chipman and Moore [2]).

It would be most convenient if there was a strong link between observable changes in national incime (according to he Laspeyres, or Paasche criterion, or both) and changes in potential welfare. However, some time ago Samuelson [12] pointed out that there were fundamental difficulties in the unqualified use of national income as an indicator of social welfare. More recently, Chipman and Moore $[3,4,6]$, have made precise the tenuous relationship between increases in national income and improvements in potential welfare. Their results can be summarized as follows:

(1) It is true that under very general conditions, an increase in national income according to the Laspeyres criterion is a necessary condition for an improvement in potential welfare in the second situation $[4$, pp. 415-416]

(2) However, an increase in national income according to both criteria is not, in general, a sufficient condition for inferring that such an improvement has taken place [4, pp. 396-398]. For this to be the case, a necessary and sufficient condition is that individual preferences should be identical and homothetic [3, pp. 160-175; and 4, pp. 403-412]

(3) When we restrict the comparison to situations in which the individual distribution of income remains constant and preferences are homothetic, the double national income test implies that potential welfare has improved only if preferences are also identical [6, pp. 409-416].

Points 2 and 3 make it clear that the notion of real national income cannot have the normative connotations that have sometimes been claimed for it. However, it could be argued that the previous tests do not rely on individual measures of welfare which take directly into account consumers preferences. Quite apart from measurement problems in practical cases, both the compensating and equivalent variations introduced by Hicks may appropriately reflect the impact on each individual of going from 
situation 1 to situation 2. Therefore, it is interesting to investigate the relationship between changes in potential welfare and the sign of either the sum of individual compensating variations (SC), or the sum of equivalent variations (SE), or both.

For a single consumer, the sign of the two measures of welfare change is necessarily the same but, in general, their magnitudes need not coincide. Consequently, SC and SE may differ in sign. Therefore, one should only study the conditions on individual preferences under which whenever we observe that SC and SE are both nonnegative (which appears to indicate that the gainers with the change can compensate the losers), it is indeed the case that the potential welfare of the community has improved.

In view of Chipman and Moore's work, if SC and SE were both nonnegative if and only if real national income raises according to both Laspeyres and Paasche criteria, we would already know the answer to the above question. As we will see below, the two double tests do not always agree on their recommendations. Nevertheless, it turns out that our results mirror faithfully those of Chipman and Moore:

(1') The nonnegativity of $\mathrm{SC}$ is a necessary condition for an improvement in potential welfare. Similarly, that SE is nonnegative is necessary condition for a deterioration in potential welfare (Theorem 1).

(2') The nonnegativity of both SC and SE guarantees that potential welfare has improved if and only if preferences are identical and homothetic (Theorems 2 and 3 ).

(3') Whenever the distribution of income remains constant and preferences are homothetic, the new double test is sufficient for a improvement in potential welfare only if preferences are identical (Theorem 4).

Boadway [1] has also written on this topic. However, he only considers a single test based on the sign of SC; his results constitute a subset of ours; he restricts himself to a diagrammatic analysis of a special economy with only two goods and two consumers; and his criteria of social welfare is the one proposed by Scitowsky which is known to be intransitive (see Gorman [8]). Therefore, we believe that to settle this question definitely, a more general and complete treatment is called for.

The paper is organized as follows. Section II presents the notation, the basic concepts, and the examples which show that a double test based on $\mathrm{SC}$ and SE is required, and that such a test is not equivalent to the one based on changes in real national income. Section III contains our results. Since the proofs consist of minor modifications of Chipman and Moore's work, they are relegated to an Appendix. The paper finishes with some concluding remarks.

\section{Notation AND Basic CONCEPTS}

1. The Kaldor-Hicks-Samuelson Ordering and Competitive Equilibria

Assume that there are $l$ consumers and $n$ commodities, and let $E^{k}$ denote the $k$-dimensional Euclidean space. An allocation $X$ is an element of $E_{+}^{l n}$, which we shall think of as an $l \times n$ matrix, whose $i$ th row, $x_{i}=\left(x_{i 1}, \ldots, x_{i n}\right)$, represents the commodity bundle received by the $i$ th agent. We use the symbol $\sigma(X)$ to denote the column sum, $\sum_{i} x_{i}$, of an allocation $X$. Given the aggregate vector of commodities $y \in E_{+}^{n}$, the attainable set (of allocations) for $y$ is defined by

$$
A(y)=\left\{X \in E_{+}^{\ln } \mid \sigma(X)=y\right\} .
$$

Each individual is assumed to have a preference ordering $R_{i}$ defined over $R^{n}$ the set of commodity bundles he can receive. Each $R_{i}$ is assumed to be a weak ordering. We shall denote by $\mathscr{R}$ the set of all weak orderings on $E_{+}^{n}$. Given an $l$-tuple $R=\left(R_{1}, \ldots, R_{l}\right) \in \mathscr{R}^{l}$ of individual preference orderings and two allocations $X$ and $X^{\prime}, X$ is said to be (weakly) Pareto superior to $X^{\prime}$ if and only if $x_{i} R_{i} x_{i}^{\prime}$ for all $i$. In this case we write $X R X^{\prime}$. Given an $l$ tuple $R \in \mathscr{R}^{l}$ and two aggregate commodity vectors $y$ and $y^{\prime}, y$ is said to be non-inferior according to the KHS criterion (or potentially superior) to $y^{\prime}$, if and only if for every allocation $X^{\prime}$ in $A\left(y^{\prime}\right)$ there exists an allocation $X$ in $A(y)$ such that $X R X^{\prime}$. In that case we write $y \geqslant_{R} y^{\prime}$

The $i$ th demand correspondence $h_{i}$ induced by $R_{i}$ assigns to every price vector $p>0$ and money income $m \geqslant 0$ the set of commodity bundles $h_{i}(p, m)=\left\{x_{i} \in E_{+}^{n} \mid\left(\forall \tilde{x}_{i} \in E_{+}^{n}\right): p \cdot \tilde{x}_{i} \leqslant m \Rightarrow x_{i} R_{i} \tilde{x}_{i}\right\}$. If $R \in \mathscr{R}^{\prime}$, the triple $(X, y, p)$ constitutes a competitive equilibrium for $R$ if and only if

$$
\begin{aligned}
& \text { (i) } p>0 \\
& \text { (ii) } x_{i} \in h_{i}\left(p, m_{i}\right) \text {, where } m_{i}=p \cdot x_{i}>0 \text { for all } i \text {; and } \\
& \text { (iii) } y=\sigma(X) \text {. }
\end{aligned}
$$

We shall denote the set of all such triples by $\xi(R)$.

The following properties of preference orderings will be used as hypotheses in the results of this paper. A preference ordering $R_{i} \in \mathscr{R}$ will be said to be

(i) nonsaturating $\left(\forall x_{i} \in E_{+}^{n}\right)\left(\exists \tilde{x}_{i} \in E_{+}^{n}\right): \tilde{x}_{i} P_{i} x_{i}$

(ii) weakly monotonic $\left(\forall x_{i}, \tilde{x}_{i} \in E_{+}^{n}\right): x_{i} \geqslant \tilde{x}_{i} \Rightarrow x_{i} R_{i} \tilde{x}_{i}$

(iii) weakly convex $\left(\forall x_{i} \in E_{+}^{n}\right): R_{i} x_{i} \equiv\left\{\tilde{x}_{i} R_{i} x_{i}\right\}$ is a convex set.

(iv) convex if $R_{i}$ is weakly convex and for every $x_{i}, \tilde{x}_{i} \in E_{+}^{n}$ such that $x_{i} P_{i} \tilde{x}_{i}$, we have: $(\lambda \in(0,1)):\left[\lambda x_{i}+(1-\lambda) \tilde{x}_{i}\right] P_{i} \tilde{x}_{i}$. 
(v) convex to the origin $\left(\forall x_{i} \in E_{+}^{n}\right)\left(\exists p \in \operatorname{int} E_{+}^{n}\right)\left(\tilde{x}_{i} \in R_{i} x_{i}\right): p \cdot \tilde{x}_{i} \geqslant$ $p \cdot x_{i}$ (see [3, Lemma 2.9, p. 157] for some implications of this assumption).

(vi) upper semi-continuous $\left(\forall x_{i} \in E_{+}^{n}\right): R_{i} x_{i}$ is a closed set.

(vii) lower semi-continuous $\left(\forall x_{i} \in E_{+}^{n}\right): x_{i} R_{i} \equiv\left\{\tilde{x}_{i} \in E_{+}^{n} \mid x_{i} R_{i} \tilde{x}_{i}\right\}$ is a closed set

(viii) continuous if $R_{i}$ is both upper- and lower-semicontinuous.

(ix) homothetic $\left(\forall x_{i}, \tilde{x}_{i} \in E_{+}^{n}\right): x_{i} R_{i} \tilde{x}_{i} \Rightarrow\left(\lambda \in E_{+}^{1}\right): \lambda x_{i} R_{i} \lambda \tilde{x}_{i}$

2. The Double Test based on the Individual Compensating and Equivalent Variations

We shall define the compensating and equivalent variations for an individual using the concept of the income-compensating function $\mu_{i}$. For that purpose, let

$$
\Omega=\left\{(p, m) \in E^{n+1} \mid p>0, m \geqslant 0\right\},
$$

and define first, for each $i$, the indirect preference relation $R_{i}^{*}$ as follows: for each $(p, m)$ and $\left(p^{\prime}, m^{\prime}\right)$ in $\Omega$

$$
(p, m) R_{i}^{*}\left(p^{\prime}, m^{\prime}\right) \Leftrightarrow h_{i}(p, m) R_{i} h_{i}\left(p^{\prime}, m^{\prime}\right) .
$$

Now, given $\left(p^{0}, m^{0}\right)$ in $\Omega$, the income-compensating function $\mu_{i}$ assigns to any positive price vector $p$ the nonnegative real number

$$
\mu_{i}\left(p ; p^{0}, m^{0}\right)=\left\{\begin{array}{lll}
\inf \left\{m>0 \mid(p, m) R_{i}^{*}\left(p^{0}, m^{0}\right)\right\} & \text { if } m^{0}>0 \\
0 & \text { if } m^{0}=0 .
\end{array}\right.
$$

The properties of this function which we shall use are the following (for a proof, see Hurwicz and Uzawa [11]):

(1) $\mu_{i}\left(p^{0} ; p^{0}, m^{0}\right)=m^{0}$

(2) If $R_{i}^{*}$ is continuous, then the function $V_{\bar{p}}$ defined for any fixed $\bar{p}$ by $V_{\bar{p}}(p, m)=\mu_{i}(\bar{p} ; p, m)$ is an indirect utility function representing $R_{i}^{*}$.

Hicks [10] introduced the compensating and equivalent variations for an individual resulting from a change in the price vector while income remained fixed. When income also changes, the compensating and equivalent variations in going from $\left(p^{1}, m_{i}^{1}\right)$ to $\left(p^{2}, m_{i}^{2}\right)$ are defined by, respectively,

$$
C_{i}=m_{i}^{2}-\mu_{i}\left(p^{2} ; p^{1}, m_{i}^{1}\right)
$$

and

$$
E_{i}=\mu_{i}\left(p^{1} ; p^{2}, m_{i}^{2}\right)-m_{i}^{1} .
$$

Properties (1) and (2) of the income compensating function ensure that for every individual

$$
C_{i} \geqslant 0 \Leftrightarrow E_{i} \geqslant 0 \Leftrightarrow\left(p^{2}, m_{i}^{2}\right) R_{i}^{*}\left(p^{1}, m_{i}^{1}\right) .
$$

At the aggregate level, if either $\mathrm{SC}=\sum_{i} C_{i}$ or $\mathrm{SE}=\sum_{i} E_{i}$ are nonnegative, one might presume that the gainers from the change should be able to compensate the losers. However, as Example 1 in Appendix I shows, SC and SE can certainly differ in sign. In view of this example, and the fact that using both SC and SE would appear to reinforce our ability to indicate correctly whether a change in social welfare has occured, what we call the variation test (VT) becomes:

(i) id SC and SE are both nonnegative: select $y^{2}$;

(ii) if SC and SE are both negative: select $y^{1}$;

(iii) otherwise: no recomendation is possible.

As Hause [9] pointed out, the compensating variation, unlike the equivalent variation, is not a proper numerical indicator of an individual's preferences when more than two alternatives are being compared. However, since our results for the double test are in the negative when there are only a pair of alternatives to choose from, transitivity need not concern us hee. In any case, our proofs will go through as well if we base the aggregate test only on the sign of SE; in fact, necessary conditions for the validity of a test based solely upon SE will follow as a special case of the necessary conditions obtained here for the validity of the double test.

3. The Lack of Equivalence with the National Income Test

By the national income test (NIT), we understand the following:

(i) if $p^{2} \cdot y^{2} \geqslant p^{2} \cdot y^{1}$ and $p^{1} \cdot y^{2} \geqslant p^{1} \cdot y^{1}$ : select $y^{2}$;

(ii) if $p^{2} \cdot y^{2}<p^{2} \cdot y^{1}$ and $p^{1} \cdot y^{2}<p^{1} \cdot y^{1}$ : select $y^{1}$;

(iii) otherwise: no recomendation is possible.

As pointed out in the Introduction, if both tests would always give rise to the same recommendations, then the fundamental results obtained by Chipman and Moore would immediately apply to the new test. However, it turns out that the two tests are not equivalent. 
The following proposition is easy to establish

Proposition 1. Suppose $R \in \mathscr{R}^{\prime}$ is an l-tuple of preference orderings, each of which is: (i) upper-semicontinuous, and (ii) convex. If $\left(X^{t}, y^{t}, p^{t}\right) \in \xi(R)$ for each $t=1,2$, then we have:

(A) $p^{2} \cdot y^{2} \geqslant p^{2} \cdot y^{1} \Rightarrow S C \geqslant 0$;

(B) $p^{1} \cdot y^{2} \leqslant p^{1} \cdot y^{1} \Rightarrow S E \leqslant 0$

Proof of Part (A). For each $i$, let $\tilde{x}_{i} \in h_{i}\left(p^{2}, \mu_{i}\left(p^{2} ; p^{1}, m_{i}^{1}\right)\right)$. Then, $x_{i}^{1} I_{i} \tilde{x}_{i}$ and, by (ii), $p^{2} \cdot x_{i}^{1} \geqslant p^{2} \cdot \tilde{x}_{i}=\mu_{i}\left(p^{2} ; p^{1}, m_{i}^{1}\right)$. Adding up for all individuals,

$$
\sum_{i} p^{2} \cdot x_{i}^{1}=p^{2} \cdot \sum_{i} x_{i}^{1}=p^{2} \cdot y^{1} \geqslant \sum_{i} p^{2} \cdot \tilde{x}_{i}=\sum_{i} \mu_{i}\left(p^{2} ; p^{1}, m_{i}^{1}\right) \text {. }
$$

Therefore,

$$
p^{2} \cdot y^{2} \geqslant p^{2} \cdot y^{1} \Rightarrow p^{2} \cdot y^{2} \geqslant \sum_{i} \mu_{i}\left(p^{2} ; p^{1}, m_{i}^{1}\right)
$$

or

$$
p^{2} \cdot y^{2}-\sum_{i} \mu_{i}\left(p^{2} ; p^{1}, m_{i}^{1}\right)=\sum_{i}\left[m_{i}^{2}-\mu_{i}\left(p^{2} ; p^{1}, m_{i}^{1}\right)\right]=\mathrm{SC} \geqslant 0
$$

Part (B) is similarly established, the argument being based on the fact that $p^{1} \times{ }_{i}^{2} \geqslant \mu_{i}\left(p^{1} ; p^{2}, m_{i}^{2}\right)$.

Q.E.D.

However, the converse of this proposition is not true: Examples 1 and 2 in Appendix I provide counterexamples to the converse of statements (A) and (B), respectively, of Proposition 1.

Once we have established that the two tests are not equivalent, it remains to consider which combination of recomendations may arise. These are the following:

(1) Both tests may recommend the selection of the same aggregate bundle as in Example 3.

(2) The NIT may select one alternative, while no recommendation is forthcoming from the VT as in Example 4.

3) The VT may select one alternative, while no recommendation is forthcoming from the NIT as in Example 2.

(4) No recommendation is forthcoming from either test as in Example 1

(5) In view of Proposition 1, it is impossible that one test recommends one of the two alternatives while the other test recommends the opposite.

\section{The Relationship Between the VT \\ and Changes in Potential Welfare}

1. Necessary Conditions for a Change in Potential Welfare We shall establish the following result:

THEOREM 1. Suppose $R \in \mathscr{R}^{\prime}$ is an l-tuple of preference orderings, each of which is (i) uper semi-continuous, and (ii) convex. If $\left(X^{t}, y^{t}, p^{t}\right) \in \xi(R)$ for $t=1,2$, then we have:

(A) $y^{2} \geqslant_{R} y^{1} \Rightarrow S C \geqslant 0$

(B) $y^{1} \geqslant_{R} y^{2} \Rightarrow S E \leqslant 0$

Proof of Part A. Let $\left(X^{t}, y^{t}, p^{t}\right) \in \xi(R), t=1,2$, and suppose $y^{2} \geqslant_{R} y^{1}$ Then there exists an allocation $\bar{X} \in A\left(y^{2}\right)$ such $a$ that $\bar{X} R X^{1}$, i.e., such that $\bar{x}_{i} R_{i} x_{i}^{1}$ for all $i$. For each $i$, let $\left.\tilde{x}_{i} \in h_{i}\left(p^{2} ; p^{1}, m_{i}^{1}\right)\right)$. Then $\bar{x}_{i} R_{i} \tilde{x}_{i}$, and, by (ii),

$$
p^{2} \cdot \bar{x}_{i} \geqslant p^{2} \cdot \bar{x}_{i} .
$$

Adding up for all consumers, we obtain

$$
\sum_{i} p^{2} \cdot \bar{x}_{i}=p^{2} \cdot \sum_{i} \bar{x}_{i}=p^{2} \cdot y^{2} \geqslant \sum_{i} p^{2} \cdot \tilde{x}_{i}=\sum_{i} \mu_{i}\left(p^{2} ; p^{1}, m_{i}^{1}\right)
$$

$$
\sum_{i}\left[m_{i}^{2}-\mu_{i}\left(p^{2} ; p^{1}, m_{i}^{1}\right)\right]=\mathrm{SC} \geqslant 0 .
$$

Part (B) is similarly established.

2. Sufficiency of the VT

That the nonnegativity of SC and SE is not sufficient for an improvement in potential welfare is demonstrated by Example 3. In that case we saw that $\mathrm{SC}=\mathrm{SE}=5$, so that $y^{2}$ is recommended over $y^{1}$ by the VT. However, recall that the competitive equilibrium allocation $X^{1}$ yields a utility level to the first individual approximately equal to 9.37 . But even if we assign to that individual the entire aggregate commodity bundle $y^{2}=(4,9)$, the maximum utility level which he can reach is 9 . Thus, it is not the case that $y^{2} \geqslant_{R} y^{1}$.

We now give sufficient conditions on individual preferences to guarantee that the nonnegativity of SC and SE implies an improvement in potential welfare. ${ }^{1}$

' Example 2 demonstrates that, even under the hypotheses of Theorem 2, the VT and the NIT are not equivalent. Therefore, Theorem 2 requires an independent proof, quite apart from Theorems 3.1 and 3.3 in Chipman and Moore [3] which provide sufficient conditions for the NIT to be valid. 
THEOREM 2. Let $R \in \mathscr{R}^{\prime}$ be an l-tuple of identical preference orderings, each of which is (i) homothetic, (ii) nonsaturating, (iii) weakly monotonic, (iv) continuous, and (v) weakly convex. Then, if $\left(X^{t}, y^{t}, p^{t}\right) \in \xi(R)$ for $t=1,2$, we have:
(A) $S C \gtreqless 0 \Leftrightarrow S E \gtreqless 0$
(B) $S C \geqslant 0 \Leftrightarrow y^{2} \geqslant_{R} y^{1}$

On the other hand, the VT is sufficient for an improvement in potential welfare only if individual preferences are identical and homothetic.

THEOREM 3. Let $R \in \mathscr{R}^{\prime}$ be an l-tuple of preference orderings, each of which is (i) continuous, and (ii) convex to the origin. If for each pair $\left(X^{1}, y^{1}, p^{1}\right),\left(X^{2}, y^{2}, p^{2}\right) \in \xi(R), S C \geqslant 0$ and $S E \geqslant 0$ together imply $y^{2} \geqslant_{R} y^{1}$ then:

(A) $R_{i}$ is homothetic for $i=1, \ldots, l$;

(B) $R_{1} \equiv R_{2} \equiv \cdots \equiv R_{\text {. }}$

3. The Case of a Fixed Income Distribution

Chipman and Moore's counterexample to the appropriatedness of the NIT as an indicator of changes in potential welfare, allows for a great variation in income distribution between the two situations being compared (see [4, pp. 396-398], or Example 3 in Appendix I). As a matter of fact, the proof that the NIT (or the VT) is valid only if preferences are identical and homothetic requires one to allow for such great variations in income distribution (see [3, Theorem 3.10])

However, as they point out, Pigou always insisted that an increase in real national income would always correctly indicate an improvement in national welfare, provided the increase referred to the aggregate income of a given group of persons with a fixed distribution of income among them. Accordingly, Chipman and Moore have investigated under what conditions on individual preferences the NIT is valid when the distribution of income is constant. They found a partial answer to this question: if preferences are homothetic, then in order for the NIT to be valid preferences must also be identical (see [6, Corollary 3.4, p. 416])

As could be expected, when one asks the same question for the VT, one finds the same partial answer. Before stating our result, we need to introduce the following concepts which are taken from [6]. Let $R \in \mathscr{R}^{\prime}$ be an $l$-tuple of preference orderings, and

$$
\delta \in S \equiv\left\{d \in E^{\prime} \mid \sum_{i} d_{i}=1, \text { and } d_{i}>0 \text { for all } i=1, \ldots, l\right\} .
$$

We define:

(a) a competitive equilibrium for $R$ and $\delta$ is a triple $(X, y, p)$ such that: (i) $p>0$; (ii) $x_{i} \in h_{i}\left(p, \delta_{i} p \cdot y\right)$ for all $i=1, \ldots, l$; and (iii) $\sigma(X)=y \geqslant 0$ We shall denote the set of all such triples by $\xi(R, \delta)$;

(b) the aggregate demand correspondence induced by $(R, \delta)$ $H(p, M ; R, \delta)$, by

$$
H(p, M ; R, \delta)=\sum_{i} h_{i}\left(p, \delta_{i} M\right) \quad \text { for } \quad(p, M) \in \Omega ;
$$

(c) the Bergson-Eisenberg social welfare ordering induced by $\delta, W_{\delta}$ on $E_{+}^{n}$ by

$$
X W_{\delta} X^{\prime} \Leftrightarrow\left[\prod_{i=1}^{l} U_{i}\left(x_{i}\right)^{\delta_{i}} \geqslant \prod_{i=1}^{\prime} U_{i}\left(x_{i}^{\prime}\right)^{\delta_{i}}\right]
$$

where, for each $i, U_{i}$ is a continuous and positively homogeneous of degree one utility function representing the preference ordering $R_{i}$;

(d) the social preference ordering induced by $\delta, R_{\delta}$, on $E_{+}^{n}$ by

$$
y R_{\delta} y^{\prime} \Leftrightarrow\left[\left(\forall X^{\prime} \in A\left(y^{\prime}\right)\right)\left(\exists X \in A(y): X W_{\delta} X^{\prime}\right)\right] ;
$$

(e) the social demand correspondence generated by $R_{\delta}, h\left(p, M, R_{\delta}\right)$

$$
\begin{gathered}
h\left(p, M ; R_{\delta}\right)=\left\{y \in E_{+}^{n} \mid p \cdot y \leqslant M, \text { and for all } y^{\prime}\right. \text { such that } \\
\left.p \cdot y^{\prime} \leqslant M \text { we have } y R_{\delta} y^{\prime}\right\}
\end{gathered}
$$

Finally, we can state the following result. ${ }^{2}$

THEOREM 4. Let $\delta \in S$ and let $R \in \mathscr{R}^{\prime}$ be a l-tuple of preference orderings, each of which is (i) continuous, (ii) convex to the origin, and (iii) homothetic. If for each pair $\left(X^{1}, y^{1}, p^{1}\right),\left(X^{2}, y^{2}, p^{2}\right) \in \xi(R, \delta), S C \geqslant 0$ and $S E \geqslant 0$ together imply $y^{2} \geqslant_{R} y^{1}$ then all individual orderings must be identical.

${ }^{2}$ As Chipman and Moore indicate, once we assume a fixed income distribution, one may concentrate on actual rather than potential welfare as a criterion of social choice. Thus, the investigate under what conditions one can guarantee that the NIT indicates correctly that $X^{2} R X^{1}$, rather than $y^{2} \geqslant_{R} y^{1}$, when the distribution of income is constant. It turns out that, again, if preferences are homothetic and satisfy some regularity conditions, then the NIT is valid in this sense only if preferences are identical. (See [6, Theorem 3.5].) We omit here the statement and proof of the corresponding result for the VT. 


\section{CONCLUDing Remarks}

The conclusion is quite clear; if we want to compare in terms of potential welfare two situations in the simplest possible case, the specific distributional effects cannot be ignored by appealing to the sign of SC and SE, except if individual preferences satisfy very strong conditions. In which case, it would be preferable to use the real national income test which only requires knowledge of price vectors and aggregate commodity bundles in the two situations under comparison. However, if we can estimate the sum of the individual compensating and equivalent variations, the following combined test would allow us to cover more situations than either the NIT or VT alone:

(i) if either the national income has risen according to both the Laspeyres and Paasche criterion, or SC and SE are both nonnegative: select $y^{2}$

(ii) if either the national income went down according to both criteria, or SC and SE are both negative: select $y^{1}$;

(iii) otherwise: no recommendation is possible.

\section{APPENDIX I}

EXAMPLE 1. Preferences are as follows: $u_{1}=x_{11}^{1 / 2} x_{12}$, and $u_{2}=x_{21} x_{22}$. The first competitive equilibrium is

$$
X^{1}=\left[\begin{array}{rr}
16 & 8 \\
8 & 2
\end{array}\right] ; \quad y^{\prime}=(24,10) ; \quad p^{1}=\left(\frac{1}{4}, 1\right)
$$

with $u_{1}^{1}=32, u_{2}^{1}=16, m_{1}^{1}=12$, and $m_{2}^{1}=4$. The second competitive equilibrium is

$$
X^{2}=\left[\begin{array}{rr}
1 & 4 \\
8 & 16
\end{array}\right] ; \quad y^{2}=(9,20) ; \quad p^{2}=(2,1)
$$

with $u_{1}^{2}=4, u_{2}^{2}=128, m_{1}^{2}=6$, and $m_{2}^{2}=32$. For any normalized price vector $p=(\rho, 1)$, the income-compensating functions are given by

$$
\mu_{1}\left(p ; u_{1}\right)=3 \rho\left(u_{1}^{2} / 4 \rho^{2}\right)^{1 / 3}
$$

and

$$
\mu_{2}\left(p ; u_{2}\right)=2\left(\rho, u_{2}\right)^{1 / 2}
$$

Therefore,

$$
\begin{aligned}
& C_{1}=m_{1}^{2}-\mu_{1}(2 ; 32)=6-24=-18, \\
& C_{2}=m_{2}^{2}-\mu_{2}(2 ; 16)=32-8 \sqrt{2}=20.7, \\
& E_{1}=\mu_{1}\left(\frac{1}{4} ; 4\right)-m_{1}^{1}=3-12=-9, \\
& E_{2}=\mu_{2}\left({ }_{4}^{1} ; 128\right)-m_{2}^{1}=11.3-4=7.3
\end{aligned}
$$

so that $\mathrm{SC}=2.7$ while $\mathrm{SE}=-1.7$. Therefore, no recommendation is possible according to the VT. Note also that $p^{2} \cdot y^{2}=38$ and $p^{2} \cdot y^{1}=58$. The fact that $\mathrm{SC}>0$ and $p^{2} \cdot y^{2}<p^{2} \cdot y^{1}$ provides a counterexample to the converse of part (A) of Proposition 1. Finally, since $p^{1} \cdot y^{2}=22.25$ and $p^{1} \cdot y^{1}=16$, no recommendation is possible according to the NIT

EXAMPLe 2. Preferences are as follows $u_{1}=x_{11} x_{12}$ and $u_{2}=x_{21} x_{22}$. The first competitive equilibrium is

$$
X^{1}=\left[\begin{array}{rr}
18 & 9 \\
2 & 1
\end{array}\right] ; \quad y^{1}=(20,10) ; \quad p^{1}=\left(\frac{1}{2}, 1\right)
$$

with $u_{1}^{1}=162, u_{2}^{1}=2, m_{1}^{1}=18$, and $m_{2}^{1}=2$. The second competitive equilibrium is:

$$
X^{2}=\left[\begin{array}{rr}
1 & 2 \\
8 & 16
\end{array}\right] ; \quad y^{2}=(9,18) ; \quad p^{2}=(2,1)
$$

with $u_{1}^{2}=2, u_{2}^{2}=128, m_{1}^{2}=4$, and $m_{2}^{2}=32$. For any normalized price vector $p=(\rho, 1)$ the income-compensating functions are

$$
\mu_{i}\left(p ; u_{i}\right)=2\left(\rho, u_{i}\right)^{1 / 2}, \quad i=1,2 .
$$

Therefore,

$$
\begin{aligned}
& E_{1}=\mu_{1}\left(\frac{1}{2} ; 2\right)-m_{1}^{1}=2-18=-16 \\
& E_{2}=\mu_{2}\left(\frac{1}{2} ; 128\right)-m_{2}^{1}=16-2=14,
\end{aligned}
$$

so that $\mathrm{SE}=-2$. But $p^{1} \cdot y^{2}=22.5>p^{1} \cdot y^{1}=20$. This provides a counterexample to the converse of part (B) of Proposition 1. Moreover $p^{2} \cdot y^{2}=36<p^{2} \cdot y^{1}=50$, and

$$
\begin{aligned}
& C_{1}=m_{1}^{2}-\mu_{1}(2 ; 162)=4-36=-32, \\
& C_{2}=m_{2}^{2}-\mu_{2}(2 ; 2)=32-4=28,
\end{aligned}
$$


so that $\mathrm{SC}=-4$. Therefore, $y^{1}$ is recommended by the VT but no recommendation is possible according to the NIT.

EXample 3 (taken from Chipman and Moore [4, p. 396]). Preferences are as follows: $u_{1}=3 \sqrt{x_{11}}+\sqrt{x_{12}}$, and $u_{2}=\sqrt{x_{21}}+3 \sqrt{x_{22}}$. The first competitive equilibrium is:

$$
X^{1}=\left[\begin{array}{ll}
8.3125 & 0.5195 \\
0.6875 & 3.4805
\end{array}\right] ; \quad y^{1}=(9,4) ; \quad p^{1}=(3,4)
$$

with $u_{1}^{1} \sim 9.37, u_{2}^{1} \sim 6.43, m_{1}^{1}=27.0155$, and $m_{2}^{1}=15.9845$. The second competitive equilibrium is:

$$
X^{2}=\left[\begin{array}{ll}
2.25 & 0.141 \\
1.75 & 8.859
\end{array}\right] ; \quad y^{2}=(4,9) ; \quad p^{2}=(3,4)
$$

with $u_{1}^{2} \sim 4.87, u_{2}^{2}=10.25, m_{1}^{2}=7.314$, and $m_{2}^{2}=40.686$. Since prices do not change, $\mu_{i}\left(p^{2} ; p^{1}, m_{i}^{1}\right)=m_{i}^{1}$ and $\mu_{i}\left(p^{1} ; p^{2}, m_{i}^{2}\right)=m_{i}^{2}$ for $i=1,2$. Therefore,

$$
\begin{aligned}
& C_{1}=E_{1}=m_{1}^{2}-m_{1}^{1}=-19.7015, \\
& C_{2}=E_{2}=m_{2}^{2}-m_{2}^{1}=24.7015,
\end{aligned}
$$

so that $\mathrm{SC}=\mathrm{SE}=5$. On the other hand

$$
p^{2} \cdot y^{2}=p^{1} \cdot y^{2}=48>p^{2} \cdot y^{1}=p^{1} \cdot y^{1}=43 \text {. }
$$

Thus $y^{2}$ is selected by both tests.

EXAMPLE 4. Preferences and income-compensating functions are as in Example 1. The first competitive equilibrium is:

$$
X^{1}=\left[\begin{array}{rr}
4 & 4 \\
16 & 8
\end{array}\right] ; \quad y^{1}=(20,12) ; \quad p^{1}=(0.5,1)
$$

with $u_{1}^{1}=8, u_{2}^{1}=128, m_{1}^{1}=6, m_{2}^{1}=16$. The second competitive equilibrium

$$
X^{2}=\left[\begin{array}{cr}
1 & 1.2 \\
18.5 & 11.1
\end{array}\right] ; \quad y^{2}=(19.5,12.3) ; \quad p^{2}=(0.6,1)
$$

with $u_{1}^{2}=1.2, \quad u_{2}^{2}=205.35, m_{1}^{2}=1.8, \quad$ and $m_{2}^{2}=22.2$. Since $p^{1} \cdot y^{2}=$ $22.5>p^{1} \cdot y^{1}=22$, and $p^{2} \cdot y^{2}=24=p^{2} \cdot y^{1}$, according to the NIT $y^{2}$ is selected over $y^{1}$. However

$$
\begin{aligned}
& C_{1}=m_{1}^{2}-\mu_{1}(0.6 ; 8) \sim-4.572, \\
& C_{2}=m_{2}^{2}-\mu_{2}(0.6 ; 128) \sim 4.67, \\
& E_{1}=\mu_{1}(0.5 ; 1.2)-m_{1}^{1} \sim-4.30, \\
& E_{2}=\mu_{2}(0.5 ; 205.35)-m_{1}^{1} \sim 4.27,
\end{aligned}
$$

so that $\mathrm{SC} \sim 0.10$, while $\mathrm{SE} \sim-0.03$. Therefore, no recommendation is possible according to the VT.

\section{APPENDIX I}

The following results will be used in the sequel. As indicated, the first three have been proved elsewhere.

Lemma 1. (A) If $R_{i} \in \mathscr{R}$ is homothetic and upper semi-contiuous, then

$$
\lambda h_{i}(p, m)=h_{i}(p, \lambda m) \quad \text { for } \quad \lambda>0
$$

(B) If, in addition, $R_{i}$ is continuous, then for any fixed $\tilde{p}$

$$
\lambda \mu_{i}(\tilde{p} ; p, m)=\mu_{i}(\tilde{p} ; \lambda m) \quad \text { for } \quad \lambda>0 .
$$

(For part (A), see [3, Lemma 2.13.i]; and for part (B), [7, Proposition H4].)

LEMma 2. Let $R \in \mathscr{R}^{\prime}$ be an l-tuple of identical preference orderings, each of which is: (i) homothetic, (ii) nonsaturating, (iii) weakly monotonic, (iv) upper-semicontinuous, and (v) weakly convex. If $\left(X^{\prime}, y^{\prime}, p^{\prime}\right) \in \xi(R)$, then for any $w \in E_{+}^{\prime}$ with $\sum_{i} w_{i}=1,\left(X^{\prime}(w), y^{\prime}, p^{\prime}\right) \in \xi(R)$, where the allocation $X^{\prime}(w)$ any $w \in E_{+}^{\prime}$ with $\sum_{i} w_{i}=1,\left(X^{\prime}(w), y^{\prime}\right.$,
is defined by $x_{i}^{\prime}(w)=w_{i} y^{\prime}$ for each $i$.

(See [3, Theorem 3.3.ii].)

Lemma 3. Let $R \in \mathscr{R}^{l}$ be an l-tuple of preference orderings each of which is: (i) nonsaturating, (ii) weakly monotonic, (iii) continuous, and (iv) homothetic. Let $\delta \in S$, and let $R_{\delta}$ be the social preference ordering induced by $\delta$. Then we have

$$
h\left(p, M ; R_{\delta}\right)=H(p, M ; R, \delta) \quad \text { for all } \quad(p, m) \in \Omega .
$$

(See [6, Lemma 2.14], proved in [5, Theorem 4.2, p. 128]) 
Lemma 4. Suppose $R \in \mathscr{R}^{\prime}$ satisfies all the hypotheses of Lemma 3, and let $\delta \in S$. Given any $(\bar{p}, \bar{M}) \in \Omega$, we have

$$
\sum_{i} \mu_{i}\left(p ; \bar{p}, \delta_{i} \bar{M}\right) \geqslant \mu(p ; \bar{p}, \bar{M}) \quad \text { for all } \quad p>0
$$

where $\mu(\cdot ; \bar{p}, M)$ is the income-compensating function associated with the social preference ordering $R_{\delta}$

Proof. Suppose, by way of contradiction, there exists some $\hat{p}>0$ such

$$
\sum \mu_{i}\left(\hat{p} ; \bar{p}, \delta_{i} \bar{M}\right)<\mu(\hat{p} ; \bar{p}, \bar{M}) .
$$

Let

$$
\hat{y} \in h\left[\hat{p}, \mu(\hat{p} ; \bar{p}, \bar{M}) ; R_{\delta}\right]
$$

and define the allocations $\tilde{X}$ and $\bar{X}$ by, respectively,

$$
\tilde{x}_{i} \in h_{i}\left[\hat{p}, \mu_{i}\left(\hat{p} ; \bar{p}, \delta_{i} \bar{M}\right)\right) \quad \text { for } \quad i=1, \ldots, l
$$

and

$$
\bar{x}_{i} \in h_{i}\left(\bar{p}, \delta_{i} \bar{M}\right) \quad \text { for } \quad i=1, \ldots, l .
$$

Then, on the one hand,

$$
\tilde{x}_{i} R_{i} \bar{x}_{i} \quad \text { for } \quad i=1, \ldots, l
$$

so that $\tilde{X}$ is at least as good as $\bar{X}$ according to the Bergson-Eisenberg social welfare function, i.e.

$$
\prod_{i=1}^{l} U_{i}\left(\tilde{x}_{i}\right)^{\delta_{i}} \geqslant \prod_{i=1}^{\prime} U_{i}\left(\bar{x}_{i}\right)^{\delta_{i}}
$$

On the other hand, if $\tilde{y}=\sigma(\tilde{X})$, we have

$$
\hat{p} \cdot \tilde{y}=\sum_{i} \hat{p} \cdot \tilde{x}_{i}=\sum_{i} \mu_{i}\left(\hat{p} ; \bar{p}, \delta_{i} \bar{M}\right) .
$$

Therefore, by (1) and (2),

$$
\hat{y} P_{\delta} \tilde{y}
$$

However,

$$
\bar{y}=\sigma(\bar{X}) \in H(\bar{p}, \bar{M} ; R, \delta)
$$

and, by Lemma 3, $\bar{y} \in h\left(\bar{p}, \bar{M} ; R_{\delta}\right)$. Therefore, $\bar{y} R_{\delta} \hat{y}$. Hence, by (4), we conclude that $\bar{y} P_{\delta} \tilde{y}$, a contradiction to (3).

Q.E.D.

\section{Proof of Theorem 2. (A) Define}

$$
w_{i}^{t}=m_{i}^{t} /\left(p^{t} \cdot y^{t}\right) \quad \text { for } \quad t=1,2 ; i=1, \ldots, l \text { so that } \sum_{i} w_{i}^{t}=1 \text { for } t=1,2 .
$$

Since preferences are identical, we have

$$
C_{i}=w_{i}^{2} p^{2} \cdot y^{2}-\mu\left(p^{2} ; p^{1}, w_{i}^{1} p^{1} \cdot y^{1}\right)
$$

and

$$
E_{i}=\mu\left(p^{1} ; p^{2}, w_{i}^{2} p^{2} \cdot y^{2}\right)-w_{i}^{1} p^{1} \cdot y^{1}
$$

for $i=1, \ldots, l$. By Lemma 1(B), income-compensating functions are homogeneous of degree one in income. Therefore, for each $i$

$$
C_{i}=w_{i}^{2} p^{2} \cdot y^{2}-w_{i}^{1} \mu\left(p^{2} ; p^{1} \cdot y^{1}\right)
$$

and

$$
E_{i}=w_{i}^{2} \mu\left(p^{1} ; p^{2}, p^{2} \cdot y^{2}\right)-w_{i}^{1} p^{1} \cdot y^{1}
$$

Adding up across consumers, we obtain

$$
\begin{aligned}
\mathrm{SC} & =p^{2} \cdot y^{2}-\mu\left(p^{2} ; p^{1}, p^{1} \cdot y^{1}\right) \\
& =\mu\left(p^{2} ; p^{2}, p^{2} \cdot y^{2}\right)-\mu\left(p^{2} ; p^{1}, p^{1} \cdot y^{1}\right) \\
& =V_{p^{2}}\left(p^{2}, p^{2} \cdot y^{2}\right)-V_{p^{2}}\left(p^{1}, p^{1} \cdot y^{1}\right),
\end{aligned}
$$

and

$$
\begin{aligned}
\mathrm{SE} & =\mu\left(p^{1} ; p^{2}, p^{2} \cdot y^{2}\right)-p^{1} \cdot y^{1} \\
& =\mu\left(p^{1} ; p^{2}, p^{2} \cdot y^{2}\right)-\mu\left(p^{1} ; p^{1}, p^{1} \cdot y^{1}\right) \\
& =V_{p^{1}}\left(p^{2}, p^{2} \cdot y^{2}\right)-V_{p^{1}}\left(p^{1}, p^{1} \cdot y^{1}\right),
\end{aligned}
$$

where $V_{p_{2}}(\cdot)$ and $V_{p_{1}}(\cdot)$ are two indirect utility functions. Hence $\mathrm{SC} \geqslant 0$ if and only if $\mathrm{SE} \geqslant 0$ as we wanted to show.

(B) By Theorem 1(A), $y^{2} \geqslant_{R} y^{1}$ implies that $\mathrm{SC} \geqslant 0$. Suppose now that $\mathrm{SC} \geqslant 0$, and let $\bar{X} \in A\left(y^{1}\right)$. If we define

$$
\tilde{x}_{i}=w_{i} y^{2}, \quad i=1, \ldots, l,
$$


where

$$
w_{i}=\mu\left(p^{2} ; p^{1}, p^{1} \cdot \bar{x}_{i}\right) / \mu\left(p^{2} ; p^{1} \cdot y^{1}\right) \quad \text { for } \quad i=1, \ldots, l ;
$$

then we have

$$
\tilde{X} \in A\left(y^{2}\right),
$$

since it follows, as in the argument developed in part (A) of the proof, that

$$
\sum_{i} \mu\left(p^{2} ; p^{1}, p^{1} \cdot \bar{x}_{i}\right)=\mu\left(p^{2} ; p^{1}, p^{1} \cdot y^{1}\right) .
$$

Furthermore, by Lemma 2 we have $\left(\tilde{X}, y^{2}, p^{2}\right) \in \xi(R)$, and therefore

$$
\tilde{x}_{i} \in h_{i}\left(p^{2}, w_{i} p^{2} \cdot y^{2}\right) \quad \text { for } \quad i=1, \ldots, l .
$$

However, since $\mathrm{SC} \geqslant 0$, we have

$$
\sum_{i}\left[m_{i}^{2}-\mu\left(p^{2} ; p^{1}, m_{i}^{1}\right)\right]=p^{2} \cdot y^{2}-\mu\left(p^{2} ; p^{1}, p^{1} \cdot y^{1}\right) \geqslant 0,
$$

and therefore

$$
\begin{aligned}
w_{i} p^{2} \cdot y^{2} & =\mu\left(p^{2} ; p^{1}, p^{1} \cdot \bar{x}_{i}\right)\left[p^{2} \cdot y^{2} / \mu\left(p^{2} ; p^{1}, p^{1} \cdot y^{1}\right)\right] \\
& \geqslant \mu\left(p^{2} ; p^{1}, p^{1} \cdot \bar{x}_{i}\right) \quad \text { for } \quad i=1, \ldots, l .
\end{aligned}
$$

Hence by (5)

$$
\tilde{x}_{i} R_{i} \bar{x}_{i} \quad \text { for } \quad i=1, \ldots, l .
$$

Since $\bar{X} \in A\left(y^{1}\right)$ was arbitrary, it follows that $y^{2} \geqslant_{R} y^{1}$.

Proof of Theorem 3. First, we show that the demand behavior of the different individuals is identical in the sense that, for all $(p, M) \in \Omega$,

$$
h_{1}(p, M)=h_{2}(p, M)=\cdots=h_{l}(p, M)=H(p, M),
$$

where $H(p, M)$ is the aggregate demand correspondence inducing by $R$.

The proof of $h_{i}(p, M) \subseteq H(p, M)$ for each $i$ is trivial, and can be found in Chipman and Moore [4, p. 404]. The proof of $H(p, M) \subseteq h_{i}(p, M)$ requires a small addition to their argument on pp. 404 406. They have $y^{2} \in H(p, M), y^{1} \in h_{1}(p, M)$ and two competitive equilibria $\left(X^{1}, y^{1}, p^{1}\right)$ and $\left(X^{2}, y^{2}, p^{2}\right)$ such that $p^{1}=p^{2}$ and $M^{1}=M^{2}$, where $M^{1}=p^{1} \cdot y^{1}$ and $M^{2}=p^{2} \cdot y^{2}$. Moreover,

$$
x_{i}^{1}= \begin{cases}y^{1} & \text { for } \quad i=1 \\ 0 & \text { for } \quad i \neq 1\end{cases}
$$

In their case, according to the NIT, $p^{2} \cdot y^{2}=p^{2} \cdot y^{1}$ and $p^{1} \cdot y^{2}=p^{1} \cdot y^{1}$ imply that $y^{2} \geqslant_{R} y^{1}$ (expression (21) on p. 405). In our case, since $p^{1}=p^{2}$, we have for each $i$,

$$
\mu_{i}\left(p^{1} ; p^{2}, m_{i}^{2}\right)=m_{i}^{2} .
$$

Therefore,

$$
\begin{aligned}
\mathrm{SE} & =\sum_{i} \mu_{i}\left(p^{1} ; p^{2}, m_{i}^{2}\right)-\sum_{i} m_{i}^{1}=\sum_{i} m_{i}^{2}-p^{1} \cdot y^{1} \\
& =p^{2} \cdot y^{2}-p^{1} \cdot y^{1}=0
\end{aligned}
$$

This, together with the fact that

$$
\begin{aligned}
\mathrm{SC} & =p^{2} \cdot y^{2}-\sum_{i} \mu_{i}\left(p^{2} ; p^{1}, m_{i}^{1}\right) \\
& =p^{2} \cdot y^{2}-\mu_{1}\left(p^{2} ; p^{1}, p^{1} \cdot y^{1}\right) \\
& =p^{2} \cdot y^{2}-\mu_{1}\left(p^{2} ; p^{2}, p^{2} \cdot y^{2}\right)=0
\end{aligned}
$$

imply also that $y^{2} \geqslant_{R} y^{1}$. From here on, the rest of their argument applies for our purposes as well.

We show next that the aggregate demand correspondence must satisfy the homogeneity property

$$
H(p, \lambda M)=\lambda H(p, M) \quad \text { for } \quad \lambda \geqslant 0 ;
$$

that is, for any $(p, M) \in \Omega$ and $\lambda \geqslant 0, \quad y \in H(p, m)$ if and only if $\lambda y \in H(p, \lambda M)$. Chipman and Moore [4] show first that the case $\lambda=0$ is trivial (p. 407). Then, they indicate that to establish (7) for $\lambda>0$, it suffices to show that

$$
\lambda H(p, M) \subseteq H(p, \lambda M)
$$

The argument to establish (8) when $\lambda$ is an integer $[4$, pp. 408-409], remains valid here. To establish (8) when $\lambda=1 / l$, they have two competitive equilibria $\left(X^{1}, y^{1}, p^{1}\right)$ and $\left(X^{2}, y^{2}, p^{2}\right)$ such that $y^{2} \in H\left(p^{2}, M^{2}\right)$;

$$
x_{i}^{2}= \begin{cases}y^{2} & \text { if } \quad i=1 \\ 0 & \text { if } \quad i \neq 1 ; \quad x_{i}^{1} \in h_{i}\left(p^{2},(1 / l) M^{2}\right) \text { for each } i=1, \ldots, l ; p^{1}-p^{2}\end{cases}
$$

and $M^{1}=M^{2}$, where $M^{1}=p^{1} \cdot y^{1}$ and $M^{2}=p^{2} \cdot y^{2}$. In their case, $p^{2} \cdot y^{2}=$ $p^{2} \cdot y^{1}$ and $p^{1} \cdot y^{2}=p^{1} \cdot y^{1}$ imply that $y^{2} \geqslant_{R} y^{1}$ (expression (43) on p. 409). 
In our case, by Proposition $1, p^{2} \cdot y^{2}=p^{2} \cdot y^{1}$ implies that $\mathrm{SC} \geqslant 0$ Moreover,

$$
\begin{aligned}
\mathrm{SE} & =\sum_{i} \mu_{i}\left(p^{1} ; p^{2}, m_{i}^{2}\right)-p^{1} \cdot y^{1} \\
& =\mu_{1}\left(p^{2} ; p^{2}, p^{2} \cdot y^{2}\right)-p^{1} \cdot y^{1}=p^{2} \cdot y^{2}-p^{1} \cdot y^{1}=0 .
\end{aligned}
$$

Thus, according to the VT, $y^{2} \geqslant_{R} y^{1}$ and the rest of their argument in p. 409-410 remains valid. To proceed from the case $\lambda=1 / l$ to $\lambda=k / l^{r}$, with $k$ and $r$ positive real numbers, is easy (see $[3$, pp. 165-166])

Finally, given (6) and (7), [3, Theorem 3.10, pp. 167-175] establishes that each $h_{i}(p, M)$ is generated by a unique preference ordering $R_{i}$ and that $R_{i}$ is homothetic.

Q.E.D

Proof of Theorem 4. First, we want to establish that for all $(p, M) \in \Omega$ and all $y \in H(p, M ; R, \delta)$ we must have $\delta_{i} y \in h_{i}\left(p, \delta_{i} M\right)$ for all $i$. For that purpose, Chipman and Moore, by way of contradiction, assume that there exist $\left(p^{0}, M^{0}\right) \in \Omega, y^{0} \in H\left(p^{0}, M^{0} ; R, \delta\right)$, and $k \in\{1, \ldots, l\}$ such that

$$
\delta_{k} y^{0} \notin h_{k}\left(p^{0}, \delta_{k} M^{0}\right) \text {. }
$$

They also construct an aggregate commodity bundle $y^{1}$ and show the existence of some $\left(p^{1}, M^{1}\right)$ satisfying the following conditions:

$$
\begin{gathered}
y^{1} \in H\left(p^{1}, M^{1} ; R, \delta\right), \\
p^{0} \cdot y^{0}=p^{0} \cdot y^{1},
\end{gathered}
$$

and

$$
y^{0} R_{\delta} y^{1}
$$

(See [6, Theorem 3.3, pp. 414 416])

Clearly, there exist allocations $X^{0} \in A\left(y^{0}\right)$ and $X^{1} \in A\left(y^{1}\right)$ such that $\left(X^{t}, y^{t}, p^{t}\right) \in \xi(R, \delta)$ for $t=0,1$. By the same argument used to establish Proposition 1(A), it follows from (9) that $\mathrm{SC} \geqslant 0$. On the other hand, let

$$
\tilde{y}^{1} \in h\left[p^{1}, \mu\left(p^{1} ; p^{0}, M^{0}\right)\right],
$$

where $\mu\left(\cdot ; p^{0}, M^{0}\right)$ is the income-compensating function associated to the social preference ordering $R_{\delta}$. Then we have $\tilde{y}^{1} R_{\delta} y^{0}$ and, in view of (10), $\tilde{y}^{1} R_{\delta} y^{1}$. Since, by Lemma $3, y^{\prime} \in h\left(p^{1}, M^{1} ; R_{\delta}\right)$, we must have

$$
p^{1} \cdot \tilde{y}^{1}=\mu\left(p^{1} ; p^{0}, M^{0}\right) \geqslant p^{1} \cdot y^{1} .
$$

Thus, by Lemma 4 ,

$$
\mathrm{SE}=\sum \mu_{i}\left(p^{1} ; p^{0}, \delta_{i} M^{0}\right)-p^{1} \cdot y^{1} \geqslant 0 .
$$

Therefore, according to the VT, we conclude that

$$
y^{0} \geqslant_{R} y^{1}
$$

which is expression (3.15) in Theorem 3.3 of $[6$, p. 415]. The rest of their argument applies here also so that, by virtue of their Lemma 3.2, one has

$$
h_{i}(p, M)=H(p, M ; R, \delta) \quad \text { for all } \quad i=1, \ldots, l .
$$

Our result follows at once from (11) and [3, Theorem 3.10, pp. 167-175] (cf. $[6$, Corollary 3.4, p. 416]).

\section{REFERENCES}

1. R. W. BosDWAy, The welfare foundations of cost-benefit analysis, Econ. J. 84 (1974) 926-939.

2. J. S. Chipman AND J. C. Moore, The compensation principle in welfare economics, in "Papers in Quantitative Economics" (A. M. Zarley, Ed.), Vo. II, pp. 1-77, Univ. of Kansa Press, Lawrence, 1971

3. J. S. Chipman AND J. C. Moore, Aggregate demand, real national income, and the compensation principle, Int. Econ. Rev. 14 (1973), 153-181

4. J. S. ChipMan and J. C. Moore, Why an increase in GNP need not imply an improvement in potential welfare, Kyklos 29 (1976), 391-418

5. J. S. Chipman AND J. C. Moore, On social welfare functions and the aggregation of preferences, J. Econ. Theory 21 (1979), 111-139.

6. J. S. CHIPMAN AND J. C. MOORE, Real national income with homothetic preferences and fixed distribution of income, Econometrica 48 (1980), 401-422.

7. J. S. ChIPMAN AND J. C. MOORE, Compensating variation, consumer's surplus, and welfare, Amer. Econ. Rev. 70 (1980), 933-949.

8. W. M. Gorman, The intransitivity of certain criteria used in welfare economics, Oxford Econ. Pap. N.S. 7 (1955), 25-35.

9. J. C. HausE, The theory of welfare cost measurement, J. Polit. Econ. 83 (1975). $1145-1182$.

10. J. R. Hicks, Consumer's surplus and index numbers, Rev. Econ. Stud. 9 (1942), 126-137. 11. L. Hurwicz AND H. Uzawa, On the integrability of demand functions, in "Preference, utility and demand" (J. S. Chipman et. al., Eds.), pp. 114-148, New York, 1971.

12. P. A. Samuelson, Evaluation of real national income, Oxford Econ. Pap. N. S. 2 (1950) $1-29$ 